\title{
EFFECT OF SAGE POWDER (SALVIA OFFICINALIS L.) ON SERUM BIOCHEMISTRY AND IMMUNITY OF BROILER CHICKS
}

\author{
() Toghyani Majid ${ }^{1}$, Akhavan Movahed Iman², Aghdam Shahryar Habib ${ }^{2}$ \\ ${ }^{1}$ Department of Animal Science, Khorasgan Branch, Islamic Azad University, Isfahan, Iran \\ ${ }^{2}$ Department of Animal Science, Shabestar Branch, Islamic Azad University, Shabestar, Iran
}

Sub-clinical application of in-feed antibiotics in animal diets has arisen into a controversial issue worldwide and is facing reduced social acceptance due to the appearance of residues and resistant strains of bacteria. Herbs, spices and various plant extracts have received increased attention as possible antibiotic growth promoter substitutions. The objective of this study was to investigate the effect of sage powder (Salvia officinalis) on serum biochemistry and immunity of broiler chicks. Two hundred and twenty five day-old broiler chicks (Ross 308 ) were randomly assigned to one of three treatments with five replicates according to a completely randomized design. The dietary treatments consisted of the basal diet as control, 4 and $8 \mathrm{~g} / \mathrm{kg}$ sage powder added to the basal diet. The broiler chicks had access to feed and water from 1 to 46 days of age. Antibody titers against Newcastle and influenza viruses (H9N2) at 26d and antibody titer against sheep red blood cell (SRBC) at 32d were measured. At $42 \mathrm{~d}$ serum were analyzed for total protein, albumin, globulin, cholesterol, high-density lipoprotein (HDL), low-density lipoprotein (LDL) cholesterol and triglyceride. Lymphoid organs (Bursa of Fabercius and spleen) also were weighed at $46 \mathrm{~d}$. The results showed antibody titer against Newcastle and Influenza viruses were elevated in broiler chicks fed $4 \mathrm{~g} / \mathrm{kg}$ sage powder $(P<0.05)$. Albumin to globulin ratio and heterophil to lymphocyte ratio were not affected by dietary treatments. Percentage of Bursa of Fabercius weight significantly increases in broilers fed $8 \mathrm{~g} / \mathrm{kg}$ sage powder $(P<0.05)$. Serum protein and triglyceride concentration were the lowest in broilers fed sage powder. Cholesterol, HDL and LDL concentrations were not affected by dietary treatments.

\section{MESENTERIC LYMPHATIC ABSORPTION AND THE PHARMACOKINETICS OF NARINGIN AND NARINGENIN IN RAT}

\section{(C) Tsai Yung-Jen ${ }^{1}$, Lin Lie-Chwen ${ }^{1,2}$, Tsai Tung-Hu ${ }^{1,3}$}

${ }^{1}$ Institute of Traditional Medicine, School of Medicine, National Yang-Ming University, Taipei, Taiwan

${ }^{2}$ National Research Institute of Chinese Medicine, Taipei, Taiwan

${ }^{3}$ Department of Education and Research, Taipei City Hospital, Taiwan

Flavonoids are a group of polyphenolic compounds with health-related properties that are widely distributed in vegetables, fruits, cocoa, teas, wines, and so on. Our hypothesis is that the flavonoids and its glycoside are absorbed mainly through the portal vein to enter the liver for biotransformation and are only partially absorbed through the lymphatic duct. The aim of study is to investigate the concentration of naringin or naringenin in the portal vein and mesenteric lymph in rats after intraduodenal administration. An unconscious, mesenteric lymphatic/portal vein/jugular vein/bile duct/duodenum- cannulated rat model was developed to investigate the partition of lymphatic and portal vein absorption. The naringin and its aglycone, naringenin, were chosen as pilot probes. Naringin was administered at dosage of 600 and $1000 \mathrm{mg} / \mathrm{kg}$, and naringenin was given at 100 and $300 \mathrm{mg} / \mathrm{kg}$. The serial concentration of naringin and naringenin recovered in mesenteric lymphatic fluid and portal blood shown that, after intraduodenal administration, both compounds were mainly absorbed into portal blood rather than mesenteric lymph. After hepatic metabolism, only a small amount of intact analyte could enter systemic circulation. Most naringin and naringenin would be eliminated through bile excretion. In conclusion, the portal and lymphatic absorption for naringin were around $95 \%$ and $5.0 \%$, respectively. The respective absorption pathways for naringenin were around $98 \%$ and $2 \%$, respectively. 